\title{
A Hybrid Lagrangian Variational Method for Bose-Einstein Condensates in Optical Lattices
}

\author{
Mark Edwards ${ }^{1} \S$, Lisa M. DeBeer ${ }^{1}$ Mads Demenikov ${ }^{1}$ Jacob \\ Galbreath $^{1}$ T. Joseph Mahaney ${ }^{1}$ Bryan Nelsen $^{1}$ and Charles \\ W. Clark ${ }^{2}$ \\ ${ }^{1}$ Department of Physics, Georgia Southern University, Statesboro, GA 30460-8031, \\ USA \\ 2 Electron and Optical Physics Division, National Institute of Standards and \\ Technology, Gaithersburg, MD 20899, USA
}

\begin{abstract}
Solving the Gross-Pitaevskii (GP) equation describing a Bose-Einstein condensate (BEC) immersed in an optical lattice potential can be a numerically demanding task. We present a variational technique for providing fast, accurate solutions of the GP equation for systems where the external potential exhibits rapid varation along one spatial direction. Examples of such systems include a BEC subjected to a one-dimensional optical lattice or a Bragg pulse. This variational method is a hybrid form of the Lagrangian Variational Method for the GP equation in which a hybrid trial wavefunction assumes a gaussian form in two coordinates while being totally unspecified in the third coordinate. The resulting equations of motion consist of a quasi-one-dimensional GP equation coupled to ordinary differential equations for the widths of the transverse gaussians. We use this method to investigate how an optical lattice can be used to move a condensate non-adiabatically.

PACS numbers: 3.75.Fi, 67.40.Db, 67.90.+Z
\end{abstract}

Submitted to: J. Phys. B: At. Mol. Phys.

$\S$ Corresponding author (edwards@georgiasouthern.edu) 


\section{Introduction}

Over the past several years, intense experimental and theoretical interest has focused on the interaction of laser light with gaseous Bose-Einstein condensates (BEC's) [1]. Examples include Bragg pulses applied to condensates and condensates confined in optical lattices 2]. Experimental and theoretical studies in these areas are important because condensates can be manipulated with Bragg pulses and strong optical lattices can confine a definite number of condensate atoms in each well. The Mott-insulator phase transition 3 ] can produce a system that might become a candidate for prototypical quantum computer.

This paper describes a method for rapidly finding accurate solutions of the GrossPitaevskii equation describing a BEC in the presence of laser light. Such cases provide some of the most demanding numerical computations in the field of gaseous BoseEinstein condensation. The "Hybrid" Lagrangian Method (HLM) described here is an instance of the Lagrangian Variational Method (LVM) 4, a method for finding approximate solutions of the Gross-Pitaevskii (GP) equation, when the condensate wavefunction exhibits rapid variation along a single spatial direction. Examples of this include Bose-Einstein condensates in optical lattices and Bragg and standing-wave laser pulses to condensates.

The method presented here is similar to several other methods presented in the literature [5]-[9]. The present method does have some significant differences. The most important difference is that the motion of the condensate transverse to the direction of the laser-light is coupled to the condensate motion along the light direction and vice versa. This coupling presents a challenge to find the correct, self-consistent, and variationally appropriate initial conditions. Such self-consistent initial conditions are not necessary in the other methods cited.

This paper is organized as follows. The equations describing the basic Lagrangian Variational Method are derived by writing down the trial wavefunction and describing the meanings of the parameters. The steps required to arrive at the final HLM equations of motion are presented. The method is then applied to the case of a BEC in the presence of an accelerated optical lattice to show that it is possible to use a lattice to move a condensate to a different location in a non-adiabatic way.

\section{The Lagrangian Variational Method}

The standard LVM is a procedure for obtaining approximate solutions of the timedependent Gross-Pitaevskii (GP) equation when a trial solution containing unknown parameters is assumed. This procedure can be summarized as follows. First, a Lagrangian density whose Euler-Lagrange equation is the GP equation is written down. Next, a trial wavefunction containing several time-dependent variational parameters is chosen. The Lagrangian whose generalized coordinates are these parameters is then obtained by inserting the trial wavefunction into the Lagrangian density and integrating. 
The time-evolution equations for these parameters are derived using the usual EulerLagrange equations for the new generalized coordinates.

It is possible to assume no particular form for the trial wavefunction and write down an Euler-Lagrange-type equation involving the Lagrangian density that yields the exact Gross-Pitaevskii partial differential equation. If one does assume a particular functional form for the trial wavefunction having time-dependent variational parameters, then equations of motion for these parameters are derived by integrating the Lagrangian density over all space yielding an ordinary Lagrangian depending only on the parameters and then applying standard Euler-Lagrange equations.

The "Hybrid" Variational Method combines these two ideas in that the trial wavefunction assumed leaves the part of the wavefunction that depends on a particular coordinate completely unspecified while assuming a particular functional form (with time-dependent variational parameters) for the part of the wavefunction depending on the other coordinates. The resulting equation of motion for the unspecified part of the wavefunction is a partial differential equation while the equations of motion for the other variational parameters are ordinary differential equation that are first-order in time.

The time-dependent GP equation for a confined condensate that is subjected to laser light has the following form.

$i \hbar \frac{\partial \psi}{\partial t}=-\frac{\hbar^{2}}{2 m} \nabla^{2} \psi+V_{\text {trap }}(\mathbf{r}, t) \psi(\mathbf{r}, t)+V_{\text {laser }}(\mathbf{r}, t) \psi(\mathbf{r}, t)+g|\psi(\mathbf{r}, t)|^{2} \psi(\mathbf{r}, t)$.

The Lagrangian density,

$$
\begin{aligned}
\mathcal{L}\left(\psi^{*}, \partial_{x} \psi^{*}, \partial_{y} \psi^{*}, \partial_{z} \psi^{*}, \partial_{t} \psi^{*} ; \mathbf{r}, t\right) & \equiv \frac{1}{2} i \hbar\left(\frac{\partial \psi^{*}}{\partial t} \psi-\psi^{*} \frac{\partial \psi}{\partial t}\right)+\frac{\hbar^{2}}{2 m}|\nabla \psi|^{2} \\
& +\left(V_{\text {trap }}(\mathbf{r}, t)+V_{\text {laser }}(\mathbf{r}, t)\right)|\psi|^{2}+\frac{1}{2} g|\psi|^{4}
\end{aligned}
$$

yields the GP equation when the variation

$$
\frac{\delta \mathcal{L}}{\delta \psi^{*}} \equiv \frac{\partial}{\partial t}\left(\frac{\partial \mathcal{L}}{\partial\left(\partial_{t} \psi^{*}\right)}\right)+\frac{\partial}{\partial x}\left(\frac{\partial \mathcal{L}}{\partial\left(\partial_{x} \psi^{*}\right)}\right)+\frac{\partial}{\partial y}\left(\frac{\partial \mathcal{L}}{\partial\left(\partial_{y} \psi^{*}\right)}\right)+\frac{\partial}{\partial z}\left(\frac{\partial \mathcal{L}}{\partial\left(\partial_{z} \psi^{*}\right)}\right)-\frac{\partial \mathcal{L}}{\partial \psi^{*}}
$$

vanishes. This is the Euler-Lagrange equation that applies when the wavefunction is completely unspecified.

To obtain a variationally approximate solution to the GP equation, we assume a trial wavefunction that contains a set of variational parameters. Each parameter is assumed to be a function of time. The most common example of a trial wavefunction is a three-dimensional gaussian: [4]

$\psi(A(t), \mathbf{w}(t), \mathbf{b}(t) ; \mathbf{r})=A(t) e^{-x^{2} / w_{x}^{2}(t)+i \beta_{x}(t) x^{2}} e^{-y^{2} / w_{y}^{2}(t)+i \beta_{y}(t) y^{2}} e^{-z^{2} / w_{z}^{2}(t)+i \beta_{z}(t) z^{2}}$

where $\mathbf{w} \equiv\left(w_{x}, w_{y}, w_{z}\right)$ and $\mathbf{b} \equiv\left(\beta_{x}, \beta_{y}, \beta_{z}\right)$.

By inserting this trial wavefunction into Eq. (3) and integrating over all space we obtain the ordinary Lagrangian:

$$
L(A(t), \mathbf{w}(t), \mathbf{b}(t))=\int \mathcal{L}[\psi(A(t), \mathbf{w}(t), \mathbf{b}(t) ; \mathbf{r})] d^{3} r .
$$


In this way, we obtain a Lagrangian in which the variational parameters are the generalized coordinates. Equations for the time dependence of these coordinates are obtained from the usual Euler-Lagrange equations. For example, for the width parameter $w_{x}$ we would have the following.

$$
\frac{d}{d t}\left(\frac{\partial L}{\partial \dot{w}_{x}}\right)-\frac{\partial L}{\partial w_{x}}=0
$$

If one carries out this procedure for the gaussian trial wavefunction given above one finds, after a little algebra, the following equation of motion for $w_{x}$

$$
\ddot{w}_{x}+\omega_{x}^{2} w_{x}=\frac{\hbar^{2}}{m^{2} w_{x}^{3}}+\sqrt{\frac{\pi}{2}} \frac{a \hbar^{2} N}{m^{2} w_{x}^{2} w_{y} w_{z}}
$$

Thus one obtains either a partial differential equation if the Euler-Lagrange equation for the Lagrangian density is used or an ordinary differential equation for a variational parameter if the integrated Lagrangian is used. The HVM combines these ideas and an example of the HVM will be derived next.

\section{The HVM equations of motion}

If a BEC is immersed in a one-dimensional optical lattice or subjected to optical laser pulses, then it is often the case that the condensate wavefunction exhibits spatial oscillations along the direction of propagation of the incident laser light that are much more rapid than along the transverse directions. In this case we can write a trial wavefunction with the following form:

$\psi\left(\phi(x, t), w_{y}(t), w_{z}(t), \beta_{y}(t), \beta_{z}(t)\right)=\phi(x, t) e^{-y^{2} / 2 w_{y}^{2}(t)+i \beta_{y}(t) y^{2}} e^{-z^{2} / 2 w_{z}^{2}(t)+i \beta_{z}(t) z^{2}}$

Notice here that the variational parameters are $\phi, w_{y}, w_{z}, \beta_{y}$, and $\beta_{z}$. We have chosen to represent the shape of the wavefunction in the transverse direction with a gaussian profile. In many cases the Thomas-Fermi approximation holds and the transverse density is more accurately represented by an inverted parabola. We shall see in section 3.3.2 that the size of the variationally determined density agrees well with the Thomas-Fermi approximation when that limit applies.

We shall assume that this trial wavefunction is normalized like this

$$
\int d^{3} r|\psi|^{2}=N
$$

where $N$ is the number of condensate atoms. The normalization condition constrains the values of the variational parameters

$$
\begin{aligned}
\int d^{3} r|\psi|^{2} & =\int_{-\infty}^{+\infty} d x|\phi(x, t)|^{2} \int_{-\infty}^{+\infty} d y e^{-y^{2} / w_{y}^{2}} \int_{-\infty}^{+\infty} d z e^{-z^{2} / w_{z}^{2}} \\
& =\left(\pi^{1 / 2} w_{y}\right)\left(\pi^{1 / 2} w_{z}\right) \int_{-\infty}^{+\infty} d x|\phi(x, t)|^{2}=N .
\end{aligned}
$$

Thus the wavefunction for the fast direction, $\phi(x, t)$, must satisfy

$$
\int_{-\infty}^{+\infty} d x|\phi(x, t)|^{2}=\frac{N}{\pi w_{y} w_{z}} .
$$

This can often be used to eliminate some variational parameters from the Lagrangian. 


\subsection{The Hybrid Lagrangian}

Since the functional dependence of the trial wavefunction on the $y$ and $z$ coordinates is assumed to be gaussian, we shall treat them in the standard LVM way. That is, we shall integrate the Lagrangian density only over these coordinates to get a "hybrid" Lagrangian

$$
L\left(\phi^{*}, \partial_{x} \phi^{*}, \partial_{t} \phi^{*}, w_{y}, w_{z}, \beta_{y}, \beta_{z}\right)=\int_{-\infty}^{+\infty} d y \int_{-\infty}^{+\infty} d z \mathcal{L}[\psi(\mathbf{r}, t)] .
$$

where we take the full Lagrangian density to be

$$
\begin{aligned}
\mathcal{L}\left(\psi^{*}, \partial_{x} \psi^{*}, \partial_{y} \psi^{*}, \partial_{z} \psi^{*}, \partial_{t} \psi^{*} ; \mathbf{r}, t\right) \equiv & \frac{1}{2} i \hbar\left(\frac{\partial \psi^{*}}{\partial t} \psi-\psi^{*} \frac{\partial \psi}{\partial t}\right)+\frac{\hbar^{2}}{2 m}|\nabla \psi|^{2} \\
& +V_{\text {ext }}(\mathbf{r}, t)|\psi|^{2}+\frac{1}{2} g|\psi|^{4}
\end{aligned}
$$

and where

$$
V_{\text {ext }}(\mathbf{r}, t)=V_{\text {trap }}(\mathbf{r}, t)+V_{\text {laser }}(x, t) .
$$

We will assume that the confining potential, $V_{\text {trap }}$, has the form of an harmonic oscillator and that the potential created by the light field, $V_{\text {laser }}$, depends only on the $x$ coordinate. The explicit forms of these are

$$
\begin{aligned}
& V_{\text {trap }}(\mathbf{r}, t)=\frac{1}{2} m \omega_{x}^{2} x^{2}+\frac{1}{2} m \omega_{y}^{2} y^{2}+\frac{1}{2} m \omega_{z}^{2} z^{2} \\
& V_{\text {laser }}(x, t)=-\hbar \frac{\Omega_{0}^{2}(t)}{2 \Delta}\left(1+\cos \left(2 k_{\mathrm{L}} x-\delta_{\mathrm{L}} t\right)\right)
\end{aligned}
$$

where $m$ is the atomic mass, $\left(\omega_{x}, \omega_{y}, \omega_{z}\right)$ are the frequencies of the harmonic confining potential, $k_{\mathrm{L}}$ is the wavevector of the laser and $\delta_{\mathrm{L}}$ is the difference in frequencies of the two counterpropagating beams that comprise the laser pulse, $\Omega_{0}$ is the single-photon Rabi frequency of the condensate atom for the laser pulse, and $\Delta$ is the detuning of the incident laser pulse from atomic resonance.

Calculating the form of the hybrid Lagrangian for the given trial wavefunction (Eq. (8) consists of (1) inserting the wavefunction into the full Lagrangian density, and (2) performing the integration over the $y$ and $z$ coordinates. We shall carry out these steps for each separate term of the full Lagrangian

$$
\begin{aligned}
\mathcal{L}_{1} & =\frac{1}{2} i \hbar\left(\frac{\partial \psi^{*}}{\partial t} \psi-\psi^{*} \frac{\partial \psi}{\partial t}\right)=\hbar \operatorname{Im}\left\{\psi^{*} \frac{\partial \psi}{\partial t}\right\} \\
\mathcal{L}_{2} & =\frac{\hbar^{2}}{2 m}|\nabla \psi|^{2}=\left(\frac{\hbar^{2}}{2 m}\right)\left(\frac{\partial \psi^{*}}{\partial x} \frac{\partial \psi}{\partial x}+\frac{\partial \psi^{*}}{\partial y} \frac{\partial \psi}{\partial y}+\frac{\partial \psi^{*}}{\partial z} \frac{\partial \psi}{\partial z}+\right) \\
\mathcal{L}_{3}=V_{\text {ext }}(\mathbf{r}, t)|\psi|^{2} & =\left(\frac{1}{2} m \omega_{x}^{2} x^{2}+\frac{1}{2} m \omega_{y}^{2} y^{2}+\frac{1}{2} m \omega_{z}^{2} z^{2}+V_{\text {laser }}(x, t)\right)|\psi|^{2} \\
\mathcal{L}_{4} & =\frac{1}{2} g|\psi|^{4}
\end{aligned}
$$

Then the resulting form of the hybrid Lagrangian for the given trial wavefunction is found from

$L_{i}\left(\phi^{*}, \partial_{x} \phi^{*}, \partial_{t} \phi^{*}, \mathbf{w}, \boldsymbol{\beta}\right)=\int_{-\infty}^{+\infty} d y \int_{-\infty}^{+\infty} d z \mathcal{L}_{i}[\psi(\mathbf{r}, t)] . \quad i=1, \ldots, 4$ 
where $\mathbf{w}=\left(w_{x}, w_{x}\right)$ and $\boldsymbol{\beta}=\left(\beta_{x}, \beta_{x}\right)$. The hybrid Lagrangian is found by inserting the trial wavefunction into the above and performing the required differentiations and integrations.

The result is

$$
\begin{aligned}
L_{\text {hybrid }}\left(\phi^{*}, \partial_{x} \phi^{*}, \partial_{t} \phi^{*}, \mathbf{w}, \boldsymbol{\beta}\right) & =\left\{\frac{\hbar}{2 i}\left(\phi^{*} \frac{\partial \phi}{\partial t}-\phi \frac{\partial \phi^{*}}{\partial t}\right)+\left(\frac{\hbar^{2}}{2 m}\right) \frac{\partial \phi^{*}}{\partial x} \frac{\partial \phi}{\partial x}+\left[\frac{1}{2} \hbar \dot{\beta}_{y} w_{y}^{2}\right.\right. \\
& +\frac{1}{2} \hbar \dot{\beta}_{z} w_{z}^{2}+\left(\frac{\hbar^{2}}{2 m}\right)\left(\frac{1}{2 w_{y}^{2}}+2 \beta_{y}^{2} w_{y}^{2}+\frac{1}{2 w_{z}^{2}}+2 \beta_{z}^{2} w_{z}^{2}\right) \\
& \left.+V_{\text {laser }}(x, t)+\frac{1}{2} m \omega_{x}^{2} x^{2}+\frac{1}{4} m \omega_{y}^{2} w_{y}^{2}+\frac{1}{4} m \omega_{z}^{2} w_{z}^{2}\right]|\phi|^{2} \\
& \left.+\frac{1}{4} g|\phi|^{4}\right\}\left(\pi^{1 / 2} w_{y}\right)\left(\pi^{1 / 2} w_{z}\right)
\end{aligned}
$$

We now present the derivation of the equations of motion for the variational parameters.

\subsection{The Euler-Lagrange equations of motion}

The next step is to derive the equations of motion for $\phi, w_{y}, w_{z}, \beta_{y}$, and $\beta_{z}$. We shall consider each in turn. We shall then discover that some further algebra will be required to make the resulting equations somewhat more convenient for calculation.

3.2.1. The equation of motion for $\phi(x, t)$ The Euler-Lagrange equation of motion for the variational parameter $\phi^{*}$ is given by

$$
\frac{\partial}{\partial t}\left(\frac{\partial L_{\text {hybrid }}}{\partial\left(\frac{\partial \phi^{*}}{\partial t}\right)}\right)+\frac{\partial}{\partial x}\left(\frac{\partial L_{\text {hybrid }}}{\partial\left(\frac{\partial \phi^{*}}{\partial x}\right)}\right)-\frac{\partial L_{\text {hybrid }}}{\partial \phi^{*}}=0 .
$$

To evaluate this equation for our case we first compute the derivatives of $L_{\text {hybrid }}$ with respect to $\phi^{*}, \partial_{t} \phi^{*}$, and $\partial_{x} \phi^{*}$. Inserting these derivatives into Eq. (19) gives the following equation of motion for $\phi$ :

$$
\begin{aligned}
i \hbar \frac{\partial \phi}{\partial t}+\frac{1}{2} i \hbar\left(\frac{\dot{w}_{y}}{w_{y}}+\frac{\dot{w}_{z}}{w_{z}}\right) \phi & =-\frac{\hbar^{2}}{2 m} \frac{\partial^{2} \phi}{\partial x^{2}}+\left(V_{\text {laser }}(x, t)+\frac{1}{2} m \omega_{x}^{2} x^{2}\right) \phi+\frac{1}{2} g|\phi|^{2} \phi \\
& +\left[\frac{1}{2} \hbar \dot{\beta}_{y} w_{y}^{2}+\frac{\hbar^{2}}{2 m}\left(\frac{1}{2 w_{y}^{2}}+2 \beta_{y}^{2} w_{y}^{2}\right)+\frac{1}{4} m \omega_{y}^{2} w_{y}^{2}\right. \\
& \left.+\frac{1}{2} \hbar \dot{\beta}_{z} w_{z}^{2}+\frac{\hbar^{2}}{2 m}\left(\frac{1}{2 w_{z}^{2}}+2 \beta_{z}^{2} w_{z}^{2}\right)+\frac{1}{4} m \omega_{z}^{2} w_{z}^{2}\right] \phi
\end{aligned}
$$

By defining

$$
\hbar f_{i}(t) \equiv \frac{1}{2} \hbar \dot{\beta}_{i} w_{i}^{2}+\frac{\hbar^{2}}{2 m}\left(\frac{1}{2 w_{i}^{2}}+2 \beta_{i}^{2} w_{i}^{2}\right)+\frac{1}{4} m \omega_{i}^{2} w_{i}^{2}, \quad i=x, y
$$

and

$$
U_{\text {ext }}(x, t) \equiv V_{\text {laser }}(x, t)+\frac{1}{2} m \omega_{x}^{2} x^{2}
$$


we can write the equation of motion for $\phi$ as

$i \hbar \frac{\partial \phi}{\partial t}+i \hbar\left(\frac{1}{2}\left[\frac{\dot{w}_{y}}{w_{y}}+\frac{\dot{w}_{z}}{w_{z}}\right]+i\left[f_{x}+f_{y}\right]\right) \phi=-\frac{\hbar^{2}}{2 m} \frac{\partial^{2} \phi}{\partial x^{2}}+U_{\text {ext }}(x, t) \phi+\frac{1}{2} g|\phi|^{2} \phi$

We can simplify this equation considerably using the following transformation:

$$
\phi(x, t)=N^{1 / 2} \tilde{\phi}(x, t) e^{-a(t)+i b(t)}
$$

where

$a(t) \equiv \frac{1}{2} \int_{0}^{t}\left[\frac{\dot{w}_{y}\left(t^{\prime}\right)}{w_{y}\left(t^{\prime}\right)}+\frac{\dot{w}_{z}\left(t^{\prime}\right)}{w_{z}\left(t^{\prime}\right)}\right] d t^{\prime} \quad$ and $\quad b(t) \equiv-\int_{0}^{t}\left(f_{y}\left(t^{\prime}\right)+f_{z}\left(t^{\prime}\right)\right) d t^{\prime}$

The result is

$$
i \hbar \frac{\partial \tilde{\phi}}{\partial t}=-\frac{\hbar^{2}}{2 m} \frac{\partial^{2} \tilde{\phi}}{\partial x^{2}}+U_{\text {ext }}(x, t) \tilde{\phi}+\frac{1}{2} g N e^{-2 a(t)}|\tilde{\phi}|^{2} \tilde{\phi}
$$

This equation can be simplified even further by noting that

$$
e^{-2 a(t)}=\frac{w_{y}(0) w_{z}(0)}{w_{y}(t) w_{z}(t)}
$$

The final form for the equation of motion for $\tilde{\phi}$ is the following

$i \hbar \frac{\partial \tilde{\phi}}{\partial t}=-\frac{\hbar^{2}}{2 m} \frac{\partial^{2} \tilde{\phi}}{\partial x^{2}}+\left(V_{\text {laser }}(x, t)+\frac{1}{2} m \omega_{x}^{2} x^{2}\right) \tilde{\phi}+\frac{1}{2} g N\left(\frac{w_{y}(0)}{w_{y}(t)}\right)\left(\frac{w_{z}(0)}{w_{z}(t)}\right)|\tilde{\phi}|^{2} \tilde{\phi}$.

This is the equation of motion for $\tilde{\phi}$. We now turn to the equations of motion for the widths and phases $w_{i}$ and $\beta_{i}$.

3.2.2. The equations of motion for the phases, $\beta_{i}$ We turn now to the Euler-Lagrange equations for the gaussian phases. These equations will provide a relationship between the phases and the gaussian widths. These relationships will be useful in simplifying the equations for the widths.

The Euler-Lagrange (EL) equations for the gaussian phases, $\beta_{y}$ and $\beta_{z}$, in terms of the hybrid Lagrangian are as follows:

$$
\frac{\partial}{\partial t}\left(\frac{\partial L_{\text {hybrid }}}{\partial \dot{\beta}_{i}}\right)-\frac{\partial L_{\text {hybrid }}}{\partial \beta_{i}}=0, \quad i=y, z .
$$

Computing the required derivatives and inserting them into the EL equation gives

$\frac{\partial}{\partial t}\left[\frac{1}{2} \hbar w_{y}^{2}|\phi(x, t)|^{2}\left(\pi^{1 / 2} w_{y}\right)\left(\pi^{1 / 2} w_{z}\right)\right]=\left[\frac{\hbar^{2}}{2 m}\right]\left(4 \beta_{y} w_{y}^{2}\right)|\phi(x, t)|^{2} \pi^{1 / 2} w_{y} \pi^{1 / 2} w_{z}$

and

$\frac{\partial}{\partial t}\left[\frac{1}{2} \hbar w_{z}^{2}|\phi(x, t)|^{2}\left(\pi^{1 / 2} w_{y}\right)\left(\pi^{1 / 2} w_{z}\right)\right]=\left[\frac{\hbar^{2}}{2 m}\right]\left(4 \beta_{z} w_{z}^{2}\right)|\phi(x, t)|^{2} \pi^{1 / 2} w_{y} \pi^{1 / 2} w_{z}$

We can simplify these equations by first considering the left-hand-side:

$$
\begin{aligned}
\frac{\partial}{\partial t}\left[\frac{1}{2} \hbar w_{y}^{2}|\phi(x, t)|^{2}\left(\pi^{1 / 2} w_{y}\right)\left(\pi^{1 / 2} w_{z}\right)\right] & =\frac{\partial}{\partial t}\left(\frac{1}{2} \hbar w_{y}^{2}\right)|\phi(x, t)|^{2}\left(\pi^{1 / 2} w_{y}\right)\left(\pi^{1 / 2} w_{z}\right) \\
& +\frac{1}{2} \hbar w_{y}^{2} \frac{\partial}{\partial t}\left[|\phi(x, t)|^{2}\left(\pi^{1 / 2} w_{y}\right)\left(\pi^{1 / 2} w_{z}\right)\right]
\end{aligned}
$$


Thus we can write the EL equation for $\beta_{y}$ as

$$
\begin{aligned}
{\left[\frac{\hbar^{2}}{2 m}\right]\left(4 \beta_{y} w_{y}^{2}\right)|\phi(x, t)|^{2} \pi^{1 / 2} w_{y} \pi^{1 / 2} w_{z} } & =\frac{\partial}{\partial t}\left(\frac{1}{2} \hbar w_{y}^{2}\right)|\phi(x, t)|^{2}\left(\pi^{1 / 2} w_{y}\right)\left(\pi^{1 / 2} w_{z}\right) \\
& +\frac{1}{2} \hbar w_{y}^{2} \frac{\partial}{\partial t}\left[|\phi(x, t)|^{2}\left(\pi^{1 / 2} w_{y}\right)\left(\pi^{1 / 2} w_{z}\right)\right]
\end{aligned}
$$

To simplify this further, multiply both sides of the above equation by $d x$ and integrate:

$$
\begin{aligned}
\left(\frac{\hbar^{2}}{2 m}\right)\left(4 \beta_{y} w_{y}^{2}\right) \int_{-\infty}^{+\infty} d x|\phi|^{2}\left(\pi^{1 / 2} w_{y}\right)\left(\pi^{1 / 2} w_{z}\right) & =\frac{\partial}{\partial t}\left(\frac{1}{2} \hbar w_{y}^{2}\right) \\
& \times \int_{-\infty}^{+\infty} d x|\phi|^{2}\left(\pi^{1 / 2} w_{y}\right)\left(\pi^{1 / 2} w_{z}\right) \\
& +\frac{1}{2} \hbar w_{y}^{2} \\
& \times \int_{-\infty}^{+\infty} d x \frac{\partial}{\partial t}\left[|\phi|^{2}\left(\pi^{1 / 2} w_{y}\right)\left(\pi^{1 / 2} w_{z}\right)\right]
\end{aligned}
$$

We assume that the derivative and the integral can be interchanged in the last term on the right. Given this to be true, we can use the normalization condition (Eq. (10))

$$
\left(\pi^{1 / 2} w_{y}\right)\left(\pi^{1 / 2} w_{z}\right) \int_{-\infty}^{+\infty} d x|\phi(x, t)|^{2}=N
$$

to show that the last term in Eq. (34) is zero and thus we have

$$
\begin{aligned}
N\left(\frac{\hbar^{2}}{2 m}\right)\left(4 \beta_{y} w_{y}^{2}\right) & =N \frac{\partial}{\partial t}\left(\frac{1}{2} \hbar w_{y}^{2}\right) \\
\left(\frac{\hbar}{m}\right)\left(4 \beta_{y} w_{y}^{2}\right) & =\frac{\partial}{\partial t}\left(w_{y}^{2}\right)=2 w_{y} \dot{w}_{y} \\
\left(\frac{\hbar}{m}\right) 2 \beta_{y} w_{y} & =\dot{w}_{y}
\end{aligned}
$$

The EL equation for $\beta_{z}$ is similar and so we finally have

$$
\begin{aligned}
& \beta_{y}=\left(\frac{m}{2 \hbar}\right) \frac{\dot{w}_{y}}{w_{y}} \\
& \beta_{z}=\left(\frac{m}{2 \hbar}\right) \frac{\dot{w}_{z}}{w_{z}}
\end{aligned}
$$

These equations can be used to eliminate $\beta_{i}$ and $\dot{\beta}_{i}(i=x, y)$ in the expressions for the $f_{i}$.

$$
\begin{aligned}
\hbar f_{i}(t) \quad & =\frac{1}{2} \hbar \dot{\beta}_{i} w_{i}^{2}+\frac{\hbar^{2}}{2 m}\left(\frac{1}{2 w_{i}^{2}}+2 \beta_{i}^{2} w_{i}^{2}\right)+\frac{1}{4} m \omega_{i}^{2} w_{i}^{2}, \\
& =\frac{1}{4} m w_{i} \ddot{w}_{i}+\frac{\hbar^{2}}{2 m} \frac{1}{2 w_{i}^{2}}+\frac{1}{4} m \omega_{i}^{2} w_{i}^{2}, \quad i=x, y .
\end{aligned}
$$

This will be useful in simplifying the equations for the gaussian widths to which we now turn. 
3.2.3. The equations of motion for the widths, $w_{i}$ We next consider the EL equations for the widths $w_{i}$, where $i=x, y$. The EL equation for the $w_{i}$ is given by

$$
\frac{\partial}{\partial t}\left(\frac{\partial L_{\text {hybrid }}}{\partial \dot{w}_{i}}\right)-\frac{\partial L_{\text {hybrid }}}{\partial w_{i}}=0 \text {. }
$$

We shall derive the equation for $w_{y}$; the derivation of the equation for $w_{z}$ is similar. Inspection shows that $L_{\text {hybrid }}$ is independent of $\dot{w}_{y}$ and so the above equation becomes

$$
\frac{\partial L_{\text {hybrid }}}{\partial w_{y}}=0 \text {. }
$$

We can easily compute this derivative:

$$
\begin{aligned}
\frac{\partial L_{\text {hybrid }}}{\partial w_{y}} & =\left\{\hbar \operatorname{Im}\left\{\phi^{*} \frac{\partial \phi}{\partial t}\right\}+\left(\frac{\hbar^{2}}{2 m}\right) \frac{\partial \phi^{*}}{\partial x} \frac{\partial \phi}{\partial x}+V_{\text {laser }}(x, t)|\phi|^{2}+\frac{1}{2} m \omega_{x}^{2} x^{2}|\phi|^{2}\right. \\
& +\frac{g}{4}|\phi|^{4}+\frac{1}{2} \hbar \dot{\beta}_{y} w_{y}^{2}|\phi|^{2}+\left(\frac{\hbar^{2}}{2 m}\right)\left(\frac{1}{2 w_{y}^{2}}+2 \beta_{y}^{2} w_{y}^{2}\right)|\phi|^{2}+\frac{1}{4} m \omega_{y}^{2} w_{y}^{2}|\phi|^{2} \\
& \left.+\frac{1}{2} \hbar \dot{\beta}_{z} w_{z}^{2}|\phi|^{2}+\left(\frac{\hbar^{2}}{2 m}\right)\left(\frac{1}{2 w_{z}^{2}}+2 \beta_{z}^{2} w_{z}^{2}\right)|\phi|^{2}+\frac{1}{4} m \omega_{z}^{2} w_{z}^{2}|\phi|^{2}\right\} \\
& \times\left(\pi^{1 / 2}\right)\left(\pi^{1 / 2} w_{z}\right) \\
& +\left\{\hbar \dot{\beta}_{y} w_{y}+\left(\frac{\hbar^{2}}{2 m}\right)\left(-\frac{1}{w_{y}^{3}}+4 \beta_{y}^{2} w_{y}\right)+\frac{1}{2} m \omega_{y}^{2} w_{y}\right\} \\
& \times\left(\pi^{1 / 2} w_{y}\right)\left(\pi^{1 / 2} w_{z}\right)|\phi|^{2}
\end{aligned}
$$

Defining

$H_{x} \equiv \hbar \operatorname{Im}\left\{\phi^{*} \frac{\partial \phi}{\partial t}\right\}+\left(\frac{\hbar^{2}}{2 m}\right) \frac{\partial \phi^{*}}{\partial x} \frac{\partial \phi}{\partial x}+V_{\text {laser }}(x, t)|\phi|^{2}+\frac{1}{2} m \omega_{x}^{2} x^{2}|\phi|^{2}+\frac{1}{4} g|\phi|^{4}$,

we can rewrite Eq. (40) as

$$
\begin{aligned}
\frac{\partial L_{\mathrm{hybrid}}}{\partial w_{y}} & =\left\{\frac{3}{2} \hbar \dot{\beta}_{y} w_{y}+\left(\frac{\hbar^{2}}{2 m}\right)\left(-\frac{1}{2 w_{y}^{3}}+6 \beta_{y}^{2} w_{y}\right)+\frac{3}{4} m \omega_{y}^{2} w_{y}\right. \\
& \left.+\frac{1}{2} \hbar \dot{\beta}_{z} \frac{w_{z}^{2}}{w_{y}}+\left(\frac{\hbar^{2}}{2 m}\right)\left(\frac{1}{2 w_{y} w_{z}^{2}}+2 \beta_{z}^{2} \frac{w_{z}^{2}}{w_{y}}\right)+\frac{1}{4} m \omega_{z}^{2} \frac{w_{z}^{2}}{w_{y}}\right\} \\
& \times\left(\pi^{1 / 2} w_{y}\right)\left(\pi^{1 / 2} w_{z}\right)|\phi|^{2}+\frac{H_{x}}{w_{y}}\left(\pi^{1 / 2} w_{y}\right)\left(\pi^{1 / 2} w_{z}\right) \\
& =0 .
\end{aligned}
$$

By multiplying by $d x$ on both sides and integrating over $x$ we obtain

$$
\begin{aligned}
\frac{\partial L_{\text {hybrid }}}{\partial w_{y}} & =N\left\{\frac{3}{2} \hbar \dot{\beta}_{y} w_{y}+\left(\frac{\hbar^{2}}{2 m}\right)\left(-\frac{1}{2 w_{y}^{3}}+6 \beta_{y}^{2} w_{y}\right)+\frac{3}{4} m \omega_{y}^{2} w_{y}\right. \\
& \left.+\frac{1}{2} \hbar \dot{\beta}_{z} \frac{w_{z}^{2}}{w_{y}}+\left(\frac{\hbar^{2}}{2 m}\right)\left(\frac{1}{2 w_{y} w_{z}^{2}}+2 \beta_{z}^{2} \frac{w_{z}^{2}}{w_{y}}\right)+\frac{1}{4} m \omega_{z}^{2} \frac{w_{z}^{2}}{w_{y}}\right\} \\
& +\pi\left\langle H_{x}\right\rangle w_{z} \\
& =0 .
\end{aligned}
$$


where

$$
\begin{aligned}
\left\langle H_{x}\right\rangle & =\int_{-\infty}^{+\infty} d x\left[\hbar \operatorname{Im}\left\{\phi^{*} \frac{\partial \phi}{\partial t}\right\}+\left(\frac{\hbar^{2}}{2 m}\right) \frac{\partial \phi^{*}}{\partial x} \frac{\partial \phi}{\partial x}+V_{\text {laser }}(x, t)|\phi|^{2}\right. \\
& \left.+\frac{1}{2} m \omega_{x}^{2} x^{2}|\phi|^{2}+\frac{g}{4}|\phi|^{4}\right]
\end{aligned}
$$

By using the equations of motion for the phases and their time derivatives we can eliminate the $\beta_{i}$ and $\dot{\beta}_{i}$ from Eq. (44)

$$
\begin{aligned}
\frac{\partial L_{\mathrm{hybrid}}}{\partial w_{y}} & =N\left\{\frac{3}{4} m \ddot{w}_{y}+\frac{1}{4} m \frac{w_{z}}{w_{y}} \ddot{w}_{z}+\left(\frac{\hbar^{2}}{2 m}\right)\left(-\frac{1}{2 w_{y}^{3}}+\frac{1}{2 w_{y} w_{z}^{2}}\right)\right. \\
& \left.+\frac{3}{4} m \omega_{y}^{2} w_{y}+\frac{1}{4} m \omega_{z}^{2} \frac{w_{z}^{2}}{w_{y}}\right\}+\pi\left\langle H_{x}\right\rangle w_{z}=0 .
\end{aligned}
$$

The equation for $w_{z}$ is obtained by writing the above equation down with $y$ and $z$ interchanged. To simplify these equations further we must consider the expression for $\left\langle H_{x}\right\rangle$.

We can express $\left\langle H_{x}\right\rangle$ in terms of $\tilde{\phi}$, defined in Eq. (24), by inserting that equation into Eq. (45) to get

$$
\left\langle H_{x}\right\rangle=N e^{-2 a(t)}\left\langle\tilde{H}_{x}\right\rangle+N e^{-2 a(t)} \hbar \dot{b}(t) \int_{-\infty}^{+\infty} d x|\tilde{\phi}|^{2}
$$

where

$$
\begin{aligned}
\left\langle\tilde{H}_{x}\right\rangle & \equiv \int_{-\infty}^{+\infty} d x\left\{\hbar \operatorname{Im}\left\{\tilde{\phi}^{*} \frac{\partial \tilde{\phi}}{\partial t}\right\}+\left(\frac{\hbar^{2}}{2 m}\right) \frac{\partial \tilde{\phi}^{*}}{\partial x} \frac{\partial \tilde{\phi}}{\partial x}\right. \\
& \left.+\left(V_{\text {laser }}(x, t)+\frac{1}{2} m \omega_{x}^{2} x^{2}\right)|\tilde{\phi}|^{2}+\frac{1}{4} g N e^{-2 a(t)}|\tilde{\phi}|^{4}\right\} .
\end{aligned}
$$

This can be significantly simplified by using the equation of motion for $\tilde{\phi}$.

$$
\left\langle\tilde{H}_{x}\right\rangle=-\int_{-\infty}^{+\infty} d x \frac{1}{4} g N e^{-2 a(t)}|\tilde{\phi}|^{4}
$$

Thus we can write a compact expression for $\left\langle H_{x}\right\rangle$. Using Eq. (47) we have

$$
\left\langle H_{x}\right\rangle=N e^{-2 a(t)}\left(-\int_{-\infty}^{+\infty} d x \frac{1}{4} g N e^{-2 a(t)}|\tilde{\phi}|^{4}-\hbar\left(f_{y}(t)+f_{z}(t)\right) \int_{-\infty}^{+\infty} d x|\tilde{\phi}|^{2}\right)
$$

Using the above expression we are at last ready to build the final equations of motion for the width parameters. The results are

$$
\begin{aligned}
& \ddot{w}_{y}+\omega_{y}^{2} w_{y}=\left(\frac{\hbar^{2}}{m^{2}}\right) \frac{1}{w_{y}^{3}}+\frac{2 \lambda}{w_{y}^{2} w_{z}} \\
& \ddot{w}_{z}+\omega_{z}^{2} w_{z}=\left(\frac{\hbar^{2}}{m^{2}}\right) \frac{1}{w_{z}^{3}}+\frac{2 \lambda}{w_{y} w_{z}^{2}},
\end{aligned}
$$

where

$$
\lambda(\tilde{\phi}) \equiv \pi w_{y}^{2}(0) w_{z}^{2}(0) \int_{-\infty}^{+\infty} d x \frac{1}{4} \frac{g N}{m}|\tilde{\phi}|^{4} .
$$

This completes the full set of equations of motion for the gaussian parameters. 
3.2.4. The final equations of motion Thus we may summarize the full set of equations of motion for this Hybrid Lagrangian Method. The trial wavefunction has the form

$\psi\left(\phi, w_{y}, w_{z}, \beta_{y}, \beta_{z}\right)=\phi(x, t) e^{-y^{2} / 2 w_{y}^{2}(t)+i \beta_{y}(t) y^{2}} e^{-z^{2} / 2 w_{z}^{2}(t)+i \beta_{z}(t) z^{2}}$.

The variational parameters are $\phi(x, t)$, the dependence of the wavefunction along the fast $(x)$ direction, $w_{y}$ and $w_{z}$, the widths of the gaussians in the transverse dimensions, and $\beta_{y}$ and $\beta_{z}$ the gaussian phases. The fast-direction wavefunction is written as

$\phi(x, t)$

$$
\begin{aligned}
& =N^{1 / 2} \tilde{\phi}(x, t) \\
& \times \exp \left\{-\frac{1}{2} \int_{0}^{t}\left[\frac{\dot{w}_{y}\left(t^{\prime}\right)}{w_{y}\left(t^{\prime}\right)}+\frac{\dot{w}_{z}\left(t^{\prime}\right)}{w_{z}\left(t^{\prime}\right)}\right] d t^{\prime}+i \int_{0}^{t}\left(f_{y}\left(t^{\prime}\right)+f_{z}\left(t^{\prime}\right)\right) d t^{\prime}\right\} \\
& \equiv N^{1 / 2} \tilde{\phi}(x, t) e^{-a(t)+i b(t)}
\end{aligned}
$$

where

$$
\begin{aligned}
\hbar f_{i}(t) \quad & =\frac{1}{2} \hbar \dot{\beta}_{i} w_{i}^{2}+\frac{\hbar^{2}}{2 m}\left(\frac{1}{2 w_{i}^{2}}+2 \beta_{i}^{2} w_{i}^{2}\right)+\frac{1}{4} m \omega_{i}^{2} w_{i}^{2}, \\
& =\frac{1}{4} m w_{i} \ddot{w}_{i}+\frac{\hbar^{2}}{2 m} \frac{1}{2 w_{i}^{2}}+\frac{1}{4} m \omega_{i}^{2} w_{i}^{2}, \quad i=x, y .
\end{aligned}
$$

and $N$ is the number of condensate atoms. The equation of motion for $\tilde{\phi}$ is

$i \hbar \frac{\partial \tilde{\phi}}{\partial t}=-\frac{\hbar^{2}}{2 m} \frac{\partial^{2} \tilde{\phi}}{\partial x^{2}}+\left(V_{\text {laser }}(x, t)+\frac{1}{2} m \omega_{x}^{2} x^{2}\right) \tilde{\phi}+\frac{1}{2} g N\left(\frac{w_{y}(0)}{w_{y}(t)}\right)\left(\frac{w_{z}(0)}{w_{z}(t)}\right)|\tilde{\phi}|^{2} \tilde{\phi}$

The equations of motion for the widths are:

$$
\begin{aligned}
& \ddot{w}_{y}+\omega_{y}^{2} w_{y}=\left(\frac{\hbar^{2}}{m^{2}}\right) \frac{1}{w_{y}^{3}}+\frac{2 \lambda}{w_{y}^{2} w_{z}} \\
& \ddot{w}_{z}+\omega_{z}^{2} w_{z}=\left(\frac{\hbar^{2}}{m^{2}}\right) \frac{1}{w_{z}^{3}}+\frac{2 \lambda}{w_{y} w_{z}^{2}},
\end{aligned}
$$

where

$$
\lambda(\tilde{\phi}) \equiv \pi w_{y}^{2}(0) w_{z}^{2}(0) \int_{-\infty}^{+\infty} d x \frac{1}{4} \frac{g N}{m}|\tilde{\phi}|^{4}
$$

Once the widths are known, the phases can be obtained from the following relations:

$$
\begin{aligned}
& \beta_{y}=\left(\frac{m}{2 \hbar}\right) \frac{\dot{w}_{y}}{w_{y}} \\
& \beta_{z}=\left(\frac{m}{2 \hbar}\right) \frac{\dot{w}_{z}}{w_{z}}
\end{aligned}
$$

Note that the equations for $\tilde{\phi}$ and the $w_{i}$ form a closed set of equations that must be solved self-consistently. Now that we have derived the equations of motion, we next consider the method for numerical solution of these equations. 
3.2.5. Scaled equations of motion To facilitate numerical solution of the HVM equations of motion it is important to cast them in scaled (dimensionless) units. The useful units of length $(\bar{d})$ and time $(1 / \bar{\omega})$ here are those of the geometrically averaged harmonic potential:

$$
\bar{d} \equiv\left(\frac{\hbar}{m \bar{\omega}}\right)^{1 / 2} \quad \bar{\omega} \equiv\left(\omega_{x} \omega_{y} \omega_{z}\right)^{1 / 3} \quad \gamma_{i} \equiv \frac{\omega_{i}}{\bar{\omega}} \quad(i=y, z)
$$

Thus the $x$ coordinate, gaussian widths, the time, and the wavefunction $\tilde{\phi}$ are scaled as follows

$\bar{x} \equiv \frac{x}{\bar{d}}, \quad \bar{w}_{i} \equiv \frac{w_{i}}{\bar{d}} \quad \bar{\beta}_{i} \equiv \frac{\bar{\beta}_{i}}{\bar{d}^{2}} \quad(i=y, z) \quad \tau \equiv \bar{\omega} t \quad \tilde{\phi} \equiv \frac{\bar{\phi}}{\bar{d}^{3 / 2}}$

Transforming the HVM equations is straightforward. The results are as follows: the scaled equations of motion consist of the psuedo-1D GP equation,

$i \frac{\partial \bar{\phi}}{\partial \tau}=-\frac{1}{2} \frac{\partial^{2} \bar{\phi}}{\partial \bar{x}^{2}}+\left(\bar{V}_{\text {laser }}(\bar{x}, \tau)+\frac{1}{2} \gamma_{x}^{2} \bar{x}^{2}\right) \bar{\phi}+\frac{1}{2}\left[4 \pi N\left(\frac{a}{\bar{d}}\right)\right]\left(\frac{\bar{w}_{y}(0)}{\bar{w}_{y}(\tau)}\right)\left(\frac{\bar{w}_{z}(0)}{\bar{w}_{z}(\tau)}\right)|\bar{\phi}|^{2} \bar{\phi},(0$

the width equations,

$$
\begin{aligned}
& \bar{w}_{y}^{\prime \prime}+\gamma_{y}^{2} \bar{w}_{y}=\frac{1}{\bar{w}_{y}^{3}}+\frac{2 \bar{\lambda}(\tau)}{\bar{w}_{y}^{2} \bar{w}_{z}} \\
& \bar{w}_{z}^{\prime \prime}+\gamma_{z}^{2} \bar{w}_{z}=\frac{1}{\bar{w}_{z}^{3}}+\frac{2 \bar{\lambda}^{\prime}(\tau)}{\bar{w}_{y} \bar{w}_{z}^{2}}
\end{aligned}
$$

where the scaled $\lambda$ parameter is

$$
\bar{\lambda}(\tau)=\frac{1}{4} \pi \bar{w}_{y}^{2}(0) \bar{w}_{z}^{2}(0)\left[4 \pi N\left(\frac{a}{\bar{d}}\right)\right] \int_{-\infty}^{+\infty} d \bar{x}|\bar{\phi}(\bar{x}, \tau)|^{4}
$$

and the scaled phase equations,

$$
\bar{\beta}_{y}=\frac{\bar{w}_{y}^{\prime}}{2 \bar{w}_{y}} \quad \text { and } \quad \bar{\beta}_{z}=\frac{\bar{w}_{z}^{\prime}}{2 \bar{w}_{z}} .
$$

These are the scaled HVM equations. The solution of these equations cannot be found unless we know the initial conditions. We next address the problem of determining these initial conditions.

\subsection{Initial conditions for the HVM equations of motion}

The initial conditions for the HVM equations are assumed to represent a Bose-Einstein condensate statically held in a magnetic trap. The proper initial values for the fastdirection wavefunction $(\phi(x, 0))$, the transverse widths $\left(w_{y}(0)\right.$ and $\left.w_{z}(0)\right)$, and the transverse phases $\left(\beta_{y}(0)\right.$ and $\left.\beta_{z}(0)\right)$ must be stationary when propagated forward in time with the HVM equations. Determining the correct initial values for these quantities is the most difficult part of solving the HVM equations because the system of equations for $\phi(x, 0), w_{y}(0)$ and $w_{z}(0)$ are nonlinearly coupled to each other and must be solved self-consistently. These equations will be developed below and an approximate analytic solution for an important special case will be derived. 
3.3.1. The equations defining the HVM initial conditions For a magnetically trapped condensate, the time evolution of the full wavefunction has the form

$$
\psi(\overline{\mathbf{r}}, \tau)=e^{-i \bar{\mu} \tau} \tilde{\psi}(\overline{\mathbf{r}}),
$$

where $\bar{\mu}$ is the scaled chemical potential. After making this transformation, the initialcondition equations are obtained by setting all of the remaining time derivatives in the HVM equations to zero:

$$
\bar{\mu} \bar{\phi}(\bar{x}, 0)=\left[-\frac{1}{2} \frac{\partial^{2}}{\partial \bar{x}^{2}}+\frac{1}{2} \gamma_{x}^{2} \bar{x}^{2}+\frac{1}{2}\left(4 \pi N\left(\frac{a}{\bar{d}}\right)\right)|\bar{\phi}(\bar{x}, 0)|^{2}\right] \bar{\phi}(\bar{x}, 0)
$$

the parameter $\bar{\mu}$ is to be determined. The equations for the widths at $\tau=0$ are

$$
\begin{aligned}
& \gamma_{y}^{2} \bar{w}_{y}(0)=\frac{1}{\bar{w}_{y}^{3}(0)}+\frac{2 \bar{\lambda}(0)}{\bar{w}_{y}^{2}(0) \bar{w}_{z}(0)} \\
& \gamma_{z}^{2} \bar{w}_{z}(0)=\frac{1}{\bar{w}_{z}^{3}(0)}+\frac{2 \bar{\lambda}(0)}{\bar{w}_{y}(0) \bar{w}_{z}^{2}(0)} .
\end{aligned}
$$

Note that the parameter $\bar{\lambda}(0)$ contains initial values of the widths and this motivates the following definition

$\bar{\lambda}(0)=\frac{1}{4} \pi \bar{w}_{y}^{2}(0) \bar{w}_{z}^{2}(0)\left[4 \pi N\left(\frac{a}{\bar{d}}\right)\right] \int_{-\infty}^{+\infty} d \bar{x}|\bar{\phi}(\bar{x}, 0)|^{4} \equiv \alpha_{0} \bar{w}_{y}^{2}(0) \bar{w}_{z}^{2}(0)$

The equations defining the initial widths then become

$$
\begin{aligned}
& \gamma_{y}^{2} \bar{w}_{y}(0)=\frac{1}{\bar{w}_{y}^{3}(0)}+2 \alpha_{0} \bar{w}_{z}(0) \\
& \gamma_{z}^{2} \bar{w}_{z}(0)=\frac{1}{\bar{w}_{z}^{3}(0)}+2 \alpha_{0} \bar{w}_{y}(0) .
\end{aligned}
$$

Equations (69) and (72) together must be solved self-consistently to obtain the initial conditions for the HVM equations. Note that, while Eq. (69) seems not to depend on the widths, this is not the case because the normalization of the full wavefunction must also be satisfied. In terms of the independent variables found in the HVM equations this becomes

$$
\int_{-\infty}^{+\infty} d \bar{x}|\bar{\phi}(\bar{x}, 0)|^{2}=\frac{1}{\pi \bar{w}_{y}(0) \bar{w}_{z}(0)}
$$

3.3.2. An approximate analytic solution For the case of a magnetic-trap potential that is axially symmetric along the fast direction and when the Thomas-Fermi limit is valid, it is possible to find an approximate analytical solution to the equations that define the proper variational initial conditions. While this solution is only approximate, it is useful because the case it covers is a common, realistic experimental situation.

When the trap potential is cylindrically symmetric, then $\gamma_{y}=\gamma_{z} \equiv \gamma_{\perp}$ and, by symmetry, it follows that

$$
\bar{w}_{y}(0)=\bar{w}_{z}(0) \equiv \bar{w}_{\perp} .
$$


In this case, the two Eqs. (72) become identical:

$$
\gamma_{\perp}^{2} \bar{w}_{\perp}=\frac{1}{\bar{w}_{\perp}^{3}}+2 \alpha_{0} \bar{w}_{\perp}
$$

As long as $2 \alpha_{0}<\gamma_{\perp}^{2}$, this simple equation has the following solution

$$
\bar{w}_{y}(0)=\bar{w}_{z}(0)=\bar{w}_{\perp}=\left(\gamma_{\perp}^{2}-2 \alpha_{0}\right)^{-1 / 4} .
$$

This is only the first step, however, since the factor $\alpha_{0}$ is not known.

The factor $\alpha_{0}$, defined as

$$
\alpha_{0} \equiv \frac{1}{4} \pi\left[4 \pi N\left(\frac{a}{\bar{d}}\right)\right] \int_{-\infty}^{+\infty} d \bar{x}|\bar{\phi}(\bar{x}, 0)|^{4},
$$

evidently depends on the fast-direction wavefunction, $\bar{\phi}(\bar{x}, 0)$, which is, as yet, unknown. A simple, approximate expression for $\bar{\phi}(\bar{x}, 0)$ can be found, in the Thomas-Fermi limit, by neglecting the kinetic-energy term in Eq. (69).

By parameterizing the chemical potential in terms of the (also unknown) condensate radius along the fast direction

$$
\bar{\mu} \equiv \frac{1}{2} \gamma_{x} \bar{x}_{0}^{2}
$$

and neglecting the kinetic energy in Eq. (69), an expression for $\bar{\phi}(\bar{x}, 0)$ can be written as

$$
\bar{\phi}(\bar{x}, 0)=\left(\frac{\frac{1}{2} \gamma_{x}^{2} \bar{x}_{0}^{2}-\frac{1}{2} \gamma_{x}^{2} \bar{x}^{2}}{\frac{1}{2}\left(4 \pi N\left(\frac{a}{d}\right)\right)}\right)^{1 / 2}, \quad|\bar{x}| \leq \bar{x}_{0}
$$

and zero otherwise. This formula can be used to obtain an expression for $\alpha_{0}$ in terms of the condensate radius, $\bar{x}_{0}$ :

$$
\alpha_{0}=\frac{\left(\frac{16}{15}\right)\left(\frac{\pi}{4}\right) \gamma_{x}^{4} \bar{x}_{0}^{5}}{\left(4 \pi N\left(\frac{a}{d}\right)\right)}
$$

Now both the initial widths and fast-direction wavefunction have been parameterized in terms of the condensate radius along the fast direction.

The value of $\bar{x}_{0}$ is determined by the normalization condition given in Eq. (73). Inserting the width and fast-direction wavefunction expressions in terms of $\bar{x}_{0}$ into the normalization condition gives an equation that determines this quantity:

$$
\bar{x}_{0}^{6}+\frac{6}{5}\left(\frac{N a}{\bar{d}}\right) \bar{x}_{0}^{5}-9\left(\frac{\gamma_{\perp}^{2}}{\gamma_{x}^{4}}\right)\left(\frac{N a}{\bar{d}}\right)^{2}=0 .
$$

By solving this equation for $\bar{x}_{0}$, an approximate initial variational wavefunction can be obtained that will be stationary when propagated using the variational equations of motion derived earlier. 


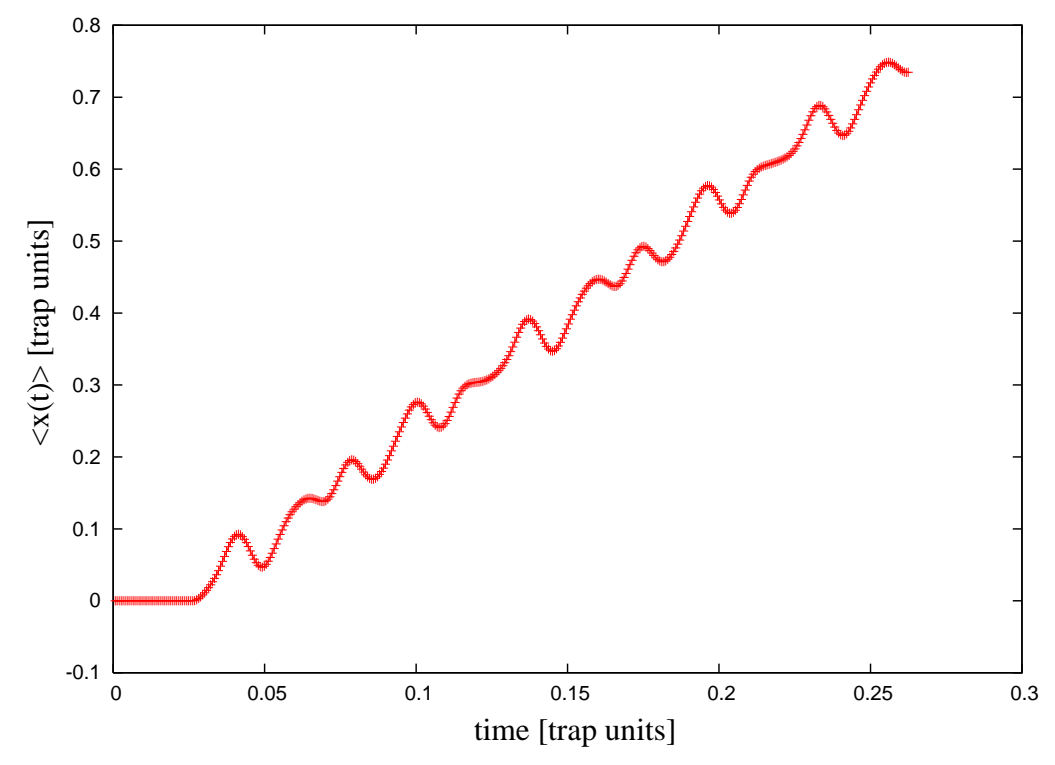

Figure 1. The average position of the condensate along the lattice direction. Here the lattice acceleration is 10 meters $/$ second $^{2}$ and the turn-on times are $T_{1}=100 \mu \mathrm{s}$ and $T_{2}=800 \mu \mathrm{s}$ (see text).

\section{Application: Moving a BEC with an optical lattice}

Finally, as an example of the usefulness of this method, it will be applied to the case of the behavior of a BEC in the presence of an accelerated optical lattice. It is possible to use an optical lattice to move a BEC from one place to another. The question arises as to whether a lattice can pick up a condensate, move it to a different location, and put it down at zero velocity. This is possible if the process is carried out completely adiabatically. However can it be accomplished rapidly? This question is considered in the following example.

The GP equation will be solved for a condensate of $N=10^{6}{ }^{87} \mathrm{Rb}$ atoms confined in a cylindrically symmetric magnetic trap with frequencies $\omega_{\perp}=(2 \pi) \times 90 \mathrm{~Hz}$ and $\omega_{z}=(2 \pi) \times 9 \mathrm{~Hz}$. A stationary optical lattice is then turned on. The lattice lasers operate at a wavelength of $\lambda=795 \mathrm{~nm}$ and its amplitude is ramped up from zero to a maximum of $5 \mathrm{E}_{\text {recoil }}$ during a timespan $T_{1}$. A standing-wave lattice can be converted into a running-wave lattice by detuning one of the counterpropagating lasers from the other one. After the lattice is ramped up to its maximum value, the lattice is accelerated and allowed to run at a maximum velocity over a time $T_{2}$ and then it is decelerated and ramped off symmetrically to the way it was turned on. The result of this sequence of

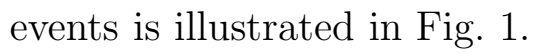

The position of the condensate is gauged by computing the average value of $x$

$\langle x\rangle=\frac{\int d^{3} r \psi^{*}(\mathbf{r}) x \psi(\mathbf{r})}{\int d^{3} r \psi^{*}(\mathbf{r}) \psi(\mathbf{r})}=\frac{\int_{-\infty}^{\infty} d x \phi^{*}(x) x \phi(x)}{\int_{-\infty}^{\infty} d x \phi^{*}(x) \phi(x)}$.

The second equality holds as a result of the assumed trial wavefunction. 
The results of this calculation are shown in Fig. 1 This figure shows that, while a stationary lattice is ramped on, the average position of the condensate is also stationary. When the lattice begins to move, the condensate is dragged along by fits and starts to a new position. These wiggles along the way are a result of the fact that the condensate is not a rigid body. When the lattice becomes stationary again its mean position moves with constant velocity but that this velocity is opposite the direction of the lattice motion. Further investigation shows that this velocity can be in either direction depending on $T_{1}$ and $T_{2}$ and that it is possible to use the lattice to pick up the condensate, move it to a new place, and put it down again with zero velocity, all done non-adiabatically. Although the condensate's final average velocity is zero, it will not be in its ground state and may be undergoing vigorous excitations. These results will be reported elsewhere.

In conclusion this paper has presented a method for rapidly finding accurate approximate solutions of the GP equation for cases where a BEC is subjected to laser light. The $3+1$ GP partial differential equation is reduced to a $1+1$ partial differential equation plus a set of three second-order ordinary differential equations in time. The latter set of equations can be solved efficiently even on a grid that can accurately represent the solution along the rapidly varying laser-light direction. This method

can be used in many applications involving condensate behavior under the influence of laser light.

\section{References}

[1] For a recent review of BEC see, Dalfovo F, et al 1999 Rev. Mod. Phys. 71 463-512; Pethick C J and Smith H 2001 Bose-Einstein Condensation in Dilute Gases (Cambridge: Cambridge University Press); Pitaevskii L P and Stingari S 2003 Bose-Einstein Condensation (Oxford: Oxford University Press).

[2] For example: Jaksch D, et al 1998 Phys. Rev. Lett. 81 3108-3111; Kozuma M, et al 1999 Phys. Rev. Lett. 82 871-875.

[3] Greiner M, et al 2002 Nature 415 39-44.

[4] Pérez-Garcia V, et al 1996 Phys. Rev. Lett. 77, 5320.

[5] Salasnich L, et al 2002 Phys. Rev. A 65, 043614.

[6] Salasnich L, et al 2002 Phys. Rev. A 66, 043603.

[7] Salasnich L, et al 2002 J. Phys. B: At. Mol. Phys. 35, 3205-3216.

[8] Salasnich L, 2000 Int. J. Mod. Phys. B 14, 1-11.

[9] Massignan M and Modugno M, 2003 Phys. Rev. A 67, 023614. 